\title{
Effective spelling training is not enhanced by tDCS in adults
}

Nathan Caruana ${ }^{1,2,3}$, Rebecca W Gelding ${ }^{1}$, Maia Zuccoํㅡ, Genevieve McArthur ${ }^{1,3}$

${ }^{1}$ Department of Cognitive Science, Macquarie University, NSW, Australia

${ }^{2}$ Perception in Action Research Centre, Macquarie University, NSW, Australia

${ }^{3}$ Macquarie University Centre for Reading, Macquarie University, NSW, Australia

Corresponding Author: Nathan Caruana' (nathan.caruana@mq.edu.au)

16 University Avenue, Macquarie University, NSW 2109, Australia

\section{Compliance with Ethical Standards}

Funding: Dr Caruana was supported by a Macquarie University Research Fellowship (Caruana MQRF). This work was also supported by the Australian Research Council (ARC) and the ARC Centre of Excellence for Cognition and its Disorders (CCD; www.ccd.edu.au) [CE110001021].

Conflict of Interest: The authors declare that they have no conflict of interest.

Ethical approval: All procedures performed in studies involving human participants were in accordance with the ethical standards of Macquarie University (see manuscript for specific approval reference numbers) and with the 1964 Helsinki declaration and its later amendments.

Informed consent: Informed consent was obtained from all individual participants included in the study.

\section{Keywords}

spelling; reading; literacy; transcranial direct current stimulation (tDCS); non-invasive brain stimulation 


\begin{abstract}
The aim of this study was to test the effects of tDCS on adult reading and spelling abilities. Experiment 1 examined whether tDCS improved adults' performance on reading and spelling tests. We found no evidence that left temporal tDCS stimulation improved word spelling, word reading accuracy, or word reading fluency compared to sham stimulation. Experiment 2 tested the effect of left-temporal tDCS stimulation on spelling training in adults. There was no evidence for a facilitatory effect of tDCS on spelling training despite its large and significant effects on adults' ability to spell difficult words. These results, paired with previous studies, argue against the use of tDCS as an intervention for poor reading and spelling until we identify a tDCS paradigm that reliably improves reading and spelling skills.
\end{abstract}


The ability to read and spell are critical for both survival and success in the modern world. Reading and spelling depend upon numerous cognitive abilities, including letter processing, conversion of letters to phonemes (or vice versa), orthographic processing, and phonological processing (Coltheart, Rastle, Perry, Langdon, \& Ziegler, 2001; Seidenberg, 2017). These cognitive skills are executed across various brain areas, including the left temporal region of the inferior frontal gyrus (Cao, Bitan, \& Booth, 2008; Landi, Mencl, Frost, Sandak, \& Pugh, 2010; Tsapkini, Vindiola, \& Rapp, 2011; Welcome \& Joanisse, 2012), the left lateral occipitotemporal region (Dehaene \& Cohen, 2011) and the left posterior superior temporal region (Graves, Grabowski, Mehta, \& Gupta, 2008). Individuals with developmental dyslexia - a condition whereby a person has unusual difficulty learning to read for no known reason - have been found to have atypical levels of activation in each of these areas (Temple et al., 2000; Eden \& Zeffiro, 1998; Pugh et al., 2000). Reading interventions that focus on word reading and spelling have been found to normalise engagement of these brain regions (Aylward et al., 2003; Simos et al., 2002).

It has been suggested that transcranial direct current stimulation (tDCS) may improve the outcomes of reading and spelling intervention (Heth \& Lavidor, 2015). tDCS is a low-cost form of noninvasive brain stimulation that places anodal and cathodal electrodes on the scalp that emit positive and negative electrical currents, respectively. This generates an electrical charge that flows through the brain. Whilst this technique cannot be used to target specific brain areas, it can be used to increase or decrease the positive charge of cells in large areas of the cortex, depending on the placement of the anodal and cathodal electrodes (Nitsche et al., 2003). In doing so, tDCS may increase or decrease the likelihood of neuronal populations reaching action potential.

In 2018, Cancer and Antonietti (2018) published a review of studies that examined the effect of tDCS on reading or spelling outcomes (see Appendix A for a summary of relevant studies). These studies can be categorised according to whether they administered (1) single tDCS stimulation before or during a reading or spelling assessment (Before: Boehringer, Macher, Dukart, Villringer, \& Pleger, 
2013; Costanzo, Varuzza, Rossi, Sdoia, Varvara, Oliveri, Koch, et al., 2016a; Cummine et al., 2019;

Heth \& Lavidor, 2015; Rios et al., 2018; Turkeltaub et al., 2012; Younger, Randazzo Wagner, \& Booth, 2016; During: Thomson et al., 2015; Westwood et al., 2017); or (2) repeated stimulation during reading or spelling training (Costanzo et al., 2016b, 2018). Most of these studies administered anodal stimulation over left-hemisphere regions which are thought to be associated with reading ability (e.g., temporo-parietal cortex, posterior temporal lobe, inferior frontal gyrus, occipital visual cortex), with cathodal or reference electrodes position over the right hemisphere (i.e., temporo-parietal cortex, orbitofrontal cortex) or on the right cheek. These studies collected control data from a sham condition or a tDCS condition that stimulated a cortical site that is not believed to be associated with reading (e.g., right superior parietal lobe, left inferior frontal gyrus).

The tDCS effects of these studies varied both within and between studies. For example, studies that examined the effect of single-session stimulation have found that left-hemispheric tDCS was associated with superior (Cummine et al., 2019; Heth and Lavidor, 2015; Turkeltaub et al., 2012; Rios et al., 2018; Younger et al., 2016), inferior (Thomson et al., 2015), or equal performance (Costanzo et al., 2016a; Westwood, 2017) on word or nonword reading assessment compared to a sham or control conditions. Further, studies that measured multiple outcomes all produced inconsistent effects of tDCS on reading or spelling assessments. This is curious given that performance on certain reading and spelling tests are reliably correlated (e.g., text reading accuracy and fluency: Costanzo et al., 2016a; word reading and nonword reading: Rios et al., 2018; word reading fluency and nonword reading fluency: Thomson et al., 2015).

Studies that used repeated stimulation during training showed more consistent results although there were only two of them - both used the same methods, and both were conducted by the same research group. Costanzo et al. (2016a and 2018) administered left anodal and right cathodal tDCS over the temporal parietal region during 18 sessions of phonics-based reading training to children $(\mathrm{N}=18)$ and adolescents $(\mathrm{N}=26)$ with developmental dyslexia. Both studies reported greater gains in 
participants' low frequency word reading accuracy or fluency, as well as and their nonword reading fluency during tDCS stimulation compared to sham.

Since Cancer and Antonietti's (2018) review, two new studies have examined the effects of repeated tDCS during reading or spelling training. In a single session, Wilson (2018) trained adults with typical development to spell 15 words that they could not spell accurately. During training they received either left anodal tDCS $(\mathrm{N}=14)$ or sham stimulation $(\mathrm{N}=14)$ over Broca's area, with cathodal stimulation to the right arm. After training, the tDCS group had higher accuracy and retention scores for trained words than the sham group. However, this study did not report whether the two groups had comparable spelling skills prior to training (Wilson, 2018). As such it is unclear whether the reported tDCS effects might reflect a confound in baseline performance.

Younger and Booth (2018) taught adults with typical reading how to read an invented orthography over a period of three days. Anodal tDCS or sham stimulation was administered to the left inferior parietal lobe in each training session. Overall, the tDCS group showed better maintenance of trained words compared to the sham group. However, this result was driven by adults whose word reading fluency was at least two standard deviations below the age-expected mean. Readers whose word reading fluency was more than two standard deviations above the mean showed reduced performance after tDCS stimulation. This is a classic example of regression-towards-the-mean, which questions the validity of the tDCS effects.

This brief summary illustrates the inconsistency of tDCS effects on reading or spelling between studies and within studies. These mixed results could arise from numerous methodological differences between studies, such as when tDCS was applied (before or during testing or training), where tDCS was applied (e.g., left temporo-parietal cortex, left inferior frontal gyrus, occipital visual cortex), the location of cathodal tDCS (e.g., right temporal-parietal cortex versus right arm), the number of tDCS sessions (e.g., 1, 3, 5 or 18), and the population sample (e.g., children or adults with or without developmental dyslexia). These mixed findings could also arise because tDCS does not have a reliable 
and real effect on reading and spelling. The aim of this study was to adjudicate between these two possibilities.

We conducted two experiments. The first used a within-subjects design to compare tDCS and sham stimulation applied to the left temporal lobe at $1.5 \mathrm{~mA}$ during reading and spelling tests in adults with typical reading and spelling ability (Experiment 1). The second experiment used a betweensubjects design to compare the effect of applying tDCS at 1.5 microamperes (mA) and sham stimulation to the left temporal lobe during eight sessions that trained adults to spell words that they could not spell accurately (Experiment 2). In both studies, we used the weight of existing evidence to design experiments that were maximally-sensitive to potential tDCS effects on reading and spelling. Specifically, we applied anodal tDCS to the left temporal lobe, which is the region most reliably associated with significant tDCS effects (Cancer \& Antonietti, 2018). We applied tDCS during, rather than before, testing and training since the weight of evidence suggests this may be more effective (see Appendix A). We recruited adults (rather than children) to minimise confounding effects of immature attention on testing or training conditions, which might attenuate any tDCS effects. We also trained spelling (rather than reading) in Experiment 2 because spelling builds more accurate orthographic representations of words, and hence may reveal more subtle tDCS effects (Conrad, Kennedy, Saoud, Scallion, \& Hanusiak, 2019). For this same reason, we trained words that adults could not spell (Wilson, 2018). We also examined the effects of tDCS at the individual level, as well as the group level, since tDCS effects are most commonly identified in studies that consider individual differences in reading abilities (Turkeltaub et al., 2012; Younger \& Booth, 2018). Finally, we chose participants with typical reading, rather than developmental dyslexia, because the latter, by definition, are resistant to literacy instruction, which could also attenuate any tDCS effects on reading or spelling. 


\section{EXPERIMENT 1}

\section{Method}

\section{Ethics}

All participants provided written and informed consent to participate in the study, which followed procedures approved by the Macquarie University (MQ) Human Research Ethics Committee (reference number: 5201400585). Participants were offered reimbursement for their time.

\section{Participants}

This study recruited 16 adult participants ( 9 females, $M_{\text {age }}=33.5, S D=9.6$, Range $=21-55$ years), who were all right-handed as confirmed by the Flinders handedness inventory (Nicholls, Thomas, Loetscher \& Grimshar, 2013), and who all passed a screening test for potential tDCS risks (see Appendix B). All participants were either completing or had completed a university degree in Australia and were Native English speakers. To control for any individual differences in literacy and intelligence we implemented a within-subjects design in which each individual served as their own, perfectly-matched control, across the tDCS and sham conditions.

\section{Measures}

To avoid test-retest effects, each participant completed a different form of a reading and spelling test during a tDCS session and a sham session, which were separated by one week. The order of treatment conditions and the type of test form was counterbalanced across participants and the exact time of day for each session were matched across sessions for each participant.

Reading fluency. Forms A and B of the Sight Word subtest of the TOWRE instruct participants to read a mixed list of increasingly-difficult regular and irregular words as quickly and accurately as possible in 45 seconds. There are no adult norms for the TOWRE, so we summed the correct number of responses to create a raw score, and then created a fluency score my multiplying the rate raw score by 45 (seconds) and dividing this by the number of seconds taken to read the words (Younger \& Booth, 2018). 
Spelling accuracy. The Green and Blue forms of the Spelling Subtest of the Wide Range Achievement Test (WRAT-4; Wilkinson \& Robertson, 2006) comprise 42 words ordered with increasing difficulty. The experimenter pronounced each word individually, read it within a sentence, and then pronounced it again. The participant was asked to write each word within 15 seconds. Testing was discontinued if the participant misspelled 10 consecutive words. Correct responses were summed and converted into standard scores provided by the WRAT-4.

Form type across participants and sessions were counterbalanced such that participants always completed form A of the TOWRE (TOWRE $\mathrm{A}$ ) and the Green form of the WRAT (WRAT $)$ in the

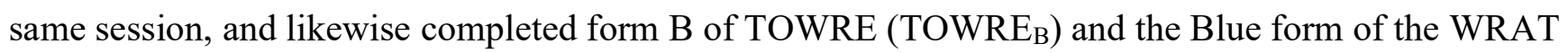
$\left(\mathrm{WRAT}_{\mathrm{B}}\right)$ in the same session. The order in which these forms were completed, and whether they were completed during tDCS or sham were evenly counterbalanced across participants.

\section{tDCS}

tDCS was administered during the reading and spelling tests outlined above. A Starstim ${ }^{\circledR}$ System (Neuroelectrics, USA) was used to stimulate the left temporal cortex using a montage of two rubber electrodes $(3 \mathrm{~cm} \times 4 \mathrm{~cm})$ placed inside cellulose sponge pockets $(5 \mathrm{~cm} \mathrm{x} 6 \mathrm{~cm})$ that were soaked in a saline solution. The centre of the stimulating (anodal) electrode was placed at T7 (10-20 system) and the centre of the return (cathodal) electrode was positioned at T8.

Stimulation started with a ramp-up period of 10 seconds, which peaked at $1.5 \mathrm{~mA}$, and then continued for 15 minutes. This is half the duration considered safe for repeated stimulation (Bikson, Datta, \& Elwassif, 2009; Fertonani, Ferrari, \& Miniussi, 2015; Nitsche et al., 2003; Poreisz, Boros, Antal, \& Paulus, 2007; Russo et al., 2013) but long enough for both the reading and spelling measures to be completed before the stimulation protocol ended. The sham stimulation started with the same 10second ramp-up period, after which stimulation was ceased (i.e., $0 \mathrm{~mA}$ ). The ramp-up period induced a physical sensation on the scalp to facilitate blinding of participants from their condition allocation. 
At the end of each session, all participants were asked to use 5-point Likert scales to rate the extent to which they experienced difficulty concentrating, relaxation, headache, fatigue, nervousness, tingling, itching, burning, or pain ( $1=$ "not at all", $5=$ "extremely"). This allowed us to detect any discomfort experienced during or after stimulation, and provided an indirect measure of whether participants were aware of their condition allocation across the two sessions (i.e., tDCS or sham).

\section{Results}

Figure 1 shows distributions of difference scores (tDCS minus sham) for TOWRE reading fluency and standardised WRAT reading accuracy scores for the different test forms. No distribution differed significantly from a normal distribution as assessed using the Shapiro-Wilk tests (all $p$ s > .759 ), and each distribution was equivariant as assessed using Levene's test (all $p \mathrm{~s}>.807$ ). Thus, parametric paired-samples t-tests were used to compare mean TOWRE reading fluency and mean WRAT spelling accuracy scores in the tDCS and sham conditions. There were no statistically significant differences between conditions for reading or spelling scores averaged across forms (see Table 1). 


\section{Facilitation Effect}

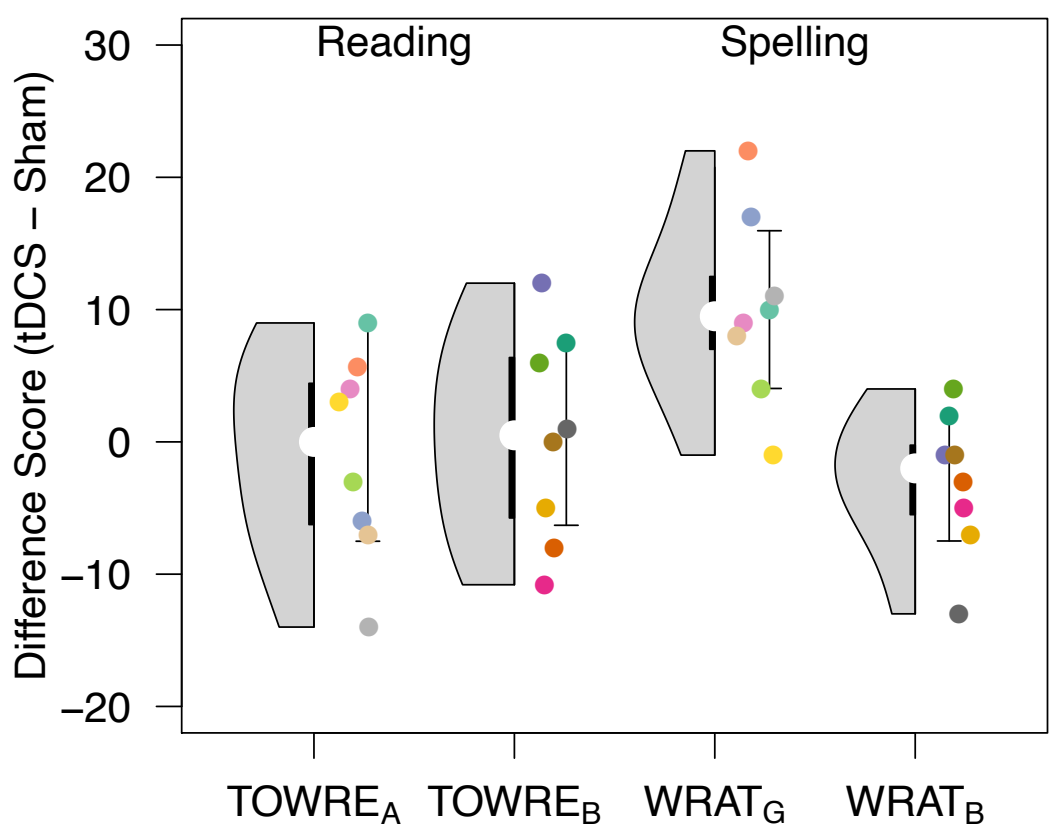

Figure 1. Difference scores between tDCS and sham conditions for TOWRE reading fluency scores (forms A and B) and WRAT spelling accuracy (forms Blue and Green). Positive scores indicate better performance during tDCS than sham. Negative scores indicate better performance during sham than tDCS. Participants who completed TOWRE $_{\mathrm{A}}$ also completed $\mathrm{WRAT}_{\mathrm{G}}$ during tDCS, whilst those who completed TOWRE $\mathrm{B}$ also completed WRAT $\mathrm{B}$ during tDCS. Coloured dots represent individual data, white dots represent group medians. Solid black lines represent the interquartile range. Whiskers represent $95 \%$ confidence intervals.

Individual data (see Figure 1) for TOWRE reading fluency revealed similar numbers of participants with higher scores during (1) tDCS compared to sham and (2) sham compared to tDCS. This was the case for both test forms. For the WRAT reading accuracy, all bar two individuals who did the Blue form during tDCS had poorer scores during tDCS than sham, while all bar one individual who did the Green form during tDCS had higher scores during tDCS than sham. In addition, the majority (78\%) of individuals who showed better spelling performance during tDCS than sham (only 56\%) did 
the WRAT Green form during tDCS, as shown in Figure 1. These observations strongly suggest form effects, which we tested statistically using independent-samples t-tests to compare participants who received tDCS during the (1) TOWRE A/WRAT Green, and (2) TOWRE B/WRAT Blue. The tDCSsham difference scores were significantly larger for participants who did the WRAT Green than those who did the WRAT Blue, while there was no significant difference between scores on the TOWRE A and B forms (see Table 1). In addition, mean scores for the WRAT Green were higher across tDCS and sham conditions than the WRAT Blue. Thus, the WRAT Green was simply easier than the WRAT Blue form.

Although we found no evidence for a tDCS effect on reading or spelling scores, we analysed participants' subjective ratings of tDCS and sham to explore whether tDCS resulted in different subjective experiences across sessions. Wilcoxon signed-rank tests revealed significantly higher ratings of 'tingling' $\left(\mathrm{M}_{\mathrm{tDCS}}=3.00, \mathrm{SD}_{\mathrm{tDCS}}=1.155 ; \mathrm{M}_{\mathrm{SHAM}}=2.125, \mathrm{SD}_{\mathrm{SHAM}}=0.957 ; W=66.5, p=.031\right)$ and 'itching' $\left(\mathrm{M}_{\mathrm{tDCS}}=2.375, \mathrm{SD}_{\mathrm{tDCS}}=1.204 ; \mathrm{M}_{\mathrm{SHAM}}=1.438, \mathrm{SD}_{\mathrm{SHAM}}=0.727 ; W=52.0, p=.013\right)$ for tDCS compared to sham (see Appendix C for all relevant statistics). There was no evidence for significant differences between treatment conditions on any other rating items (all $p \mathrm{~s}>.072$ ).

\section{Discussion}

Experiment 1 produced no evidence to suggest that left temporal tDCS improves reading fluency or spelling accuracy performance in adults. There were no statistically significant differences between the mean scores for the tDCS and sham sessions for any of the tests. Inspection of individual differences also found no effect of tDCS on reading or spelling performance. It also suggested that individuals who showed some evidence of better spelling performance under tDCS may actually be exhibiting a form effect rather than a true effect of tDCS. This was confirmed by analyses revealing that participants who did the WRAT Green during tDCS had larger tDCS-sham difference scores than those who did the WRAT Blue. In a nutshell, the WRAT Green was easier than the WRAT Blue, which created a faux tDCS effect if the WRAT Green was administered during tDCS. This result highlights a 
particular challenge for investigating the effect of single-session tDCS on cognitive skills. Not only do alternative forms of reading and spelling tests have to be perfectly matched in difficulty, the forms need to be matched for individual differences in word knowledge. More specifically, a person, or group of people (e.g., children taking the same reading class), may happen to know more words in one form but not on the other, creating differences in form difficulty.

The absence of an effect of tDCS in Experiment 1 provides direct support for two previous studies that tested the effect of tDCS applied during a reading fluency or spelling accuracy assessment (Thomson et al., 2015; Westwood et al., 2017), and indirect support for three studies which applied tDCS before assessments (Boehringer et al., 2913; Constanzo et al., 2016a; Rios et al., 2018). Together, these studies challenge the idea that one-off tDCS can facilitate performance on reading or spelling tasks. However, the results of these studies do not speak to whether tDCS might facilitate the learning of new words. This could be tested by comparing the effects of tDCS and sham stimulation during spelling training sessions on spelling accuracy. This was the goal of Experiment 2, which used a between-subjects design that also eliminated the need for alternative test forms.

\section{EXPERIMENT 2}

\section{Method}

\section{Ethics}

These were the same as Experiment 1 except for the ethics approval reference number: 5201500837.

\section{Participants}

Participants were 10 adults ( 6 females, $M_{\text {age }}=30.9, S D=6.5$, Range $=24-45$ years) with no known history of developmental difficulties. Like Experiment 1, all participants were right-handed and were screened for any potential risk of being administered tDCS (see Appendix B). They were also screened for their spelling, reading, spoken word retrieval, and non-verbal intelligence to allow us to confirm equivalence between groups. Prior to the start of the experiment, participants were randomly 
allocated to a tDCS group or a sham group. These two groups did not differ with respect to their mean age or any of the screened skills (see Table 2 and Measures below).

\section{Table 2}

Screening measures of spelling, reading, non-verbal intelligence, and spoken word retrieval.

\begin{tabular}{lrrr}
\hline Measures & \multicolumn{2}{c}{ Sham } & \multicolumn{2}{c}{ tDCS } & \multicolumn{1}{c}{$\boldsymbol{p}$} \\
\hline TOWRE Word Fluency & $97.61(23.37)$ & $103.41(7.80)$ & $0.526, .613$ \\
TOWRE Nonword Fluency & $53.80(7.79)$ & $60.40(1.52)$ & $1.859, .100^{\mathrm{a}}$ \\
Graded Naming Test & $14.00(6.34)$ & $17.60(7.54)$ & $0.816, .438$ \\
KBIT-2 Matrices & $115.40(16.97)$ & $114.60(13.24)$ & $0.083, .936$ \\
\hline
\end{tabular}

Note. Descriptive statistics are reported in the format M(SD).

a Levene's test indicated unequal variance between groups for this comparison, and thus was also tested using a Mann-Whitney non-parametric test $(p=.207)$.

\section{Design}

This experiment included three test sessions, one training period, and one no-training period. In the first test session (Test 1), participants were administered a spelling accuracy test (the primary outcome; see below) and the screening tests (see Table 2). They then completed eight spelling training sessions that focused on words that they failed to spell accurately in the Test 1 spelling accuracy test. They were retested for their spelling accuracy immediately after training had been completed (Test 2), and then after a two-week period of no training (Test 3). In total, participants attended 11 in-person sessions (see Appendix D for a visual summary of the trial design and schedule).

Tests 1, 2 and 3 were carried out by author MZ, who was blind to participants' condition allocation. Author NC carried out blind condition allocation, selection of spelling training items for each session, and administered the spelling training. Sessions were scheduled to ensure that the intervening time between test and training sessions were identical between subjects and condition groups (see Figure 2). Testing and training sessions were conducted at the same time of day for each participant. 


\section{Measures}

Spelling. The Macquarie University Advanced Adult Spelling Test (MAAST) was used to assess spelling accuracy (Caruana, Colenbrander, \& McArthur, 2019; see www.motif.com.au for the test and information about its development). The MAAST presents 72 items, in randomised order, that are very difficult to spell in English (e.g., miscellaneous, haemorrhage). Participants are asked to write each word after it has been pronounced twice by the experimenter. Words were not read within a sentence. The total number of correctly spelled words was converted into a percentage. In addition, we used the Davis Match Calculator (Davis \& Bowers, 2006) to quantify the degree to which a participant's spelling of each word matched the accurate spelling of each word. A match of 1 indicated that all letters were accurate and in the correct relative position. A match score of 0 indicated that either none of the letters were correct or none were in the correct order. We then calculated the mean match score for each individual (i.e., averaged across all 72 words in the MAAST).

Reading. The Sight Word and Nonword subtests of the TOWRE were administered at Test 1 to screen participants for their word and nonword reading ability. This is described above under Experiment 1.

Spoken language. The Graded Naming Test (McKenna \& Warrington, 1980) was used to screen for spoken word retrieval. Participants were asked to name 30 objects illustrated in black and white line drawings (e.g., a kangaroo). Corrected responses are summed to produce a total score of correctly named items.

Non-verbal intelligence. The Matrices subtest of the Kaufman Brief Intelligence Test-Second Edition (KBIT-2; Kaufman \& Kaufman, 2004) was used as a screening test for non-verbal intelligence. It presents up to 46 increasingly-difficult items that comprise a visual pattern with a missing portion. Participants are asked to choose the missing portion from a suite of options. Testing is discontinued after four consecutive incorrect responses. The total number of correct items is converted in a standard score that has a mean of 100 and standard deviation of 15. 


\section{Spelling training}

Stimuli. Prior to the first training session, each MAAST word that a participant spelled incorrectly in Test 1 was printed onto a card in lower-case Calibri font. These cards were then stacked in order of difficulty and then dealt into piles of five cards so that each pile contained an equal mix of easy and difficult words. Each pile represented a training set that was administered in a single training session.

Procedure. At the start of each training session, the instructor (NC) placed the tDCS device on the participant (see below for tDCS protocol). Once stimulation had begun, participants in both tDCS and Sham groups were asked to rest for five minutes to ensure that any initial sensations dissipated before the training. The instructor placed a small whiteboard, whiteboard marker, and eraser in front of the participant, and sat on the opposite side of the table. Then the highly-structured training instruction began, which included five training phases per session. These phases were developed from evidencebased training procedures used at the Macquarie University Reading Clinic (Kohnen \& Banales, 2015).

Training 1 (copy). The instructor shuffled the set of flashcards assigned to the training session. They showed the participant one of the flashcards and said "This word is [pronunciation of item]. Can you say this word?". If the participant read the word correctly, which was always the case, the instructor said "Well done. Now copy the word down onto the whiteboard." If the participant copied the word correctly, the instructor proceeded to the next training phase. If the participant made an error, the instructor crossed out the response, wrote the correct spelling, and explained: "What you wrote is not quite right. Look at this spelling. This is the correct spelling of the word." The participant then copied the written word.

Training 2 (delayed copy). The instructor removed the flashcard, cleaned the whiteboard, and instructed the participant to "visualise the word." After 10 seconds (timed with a stopwatch), they ask the participant to "write down the word again." If the participant correctly spelled the word, the 
instructor provided positive feedback and proceeded to train the next word in the list using the procedure outlined in 'Training 1' above. If the participant made an error, the instructor crossed out the response, wrote the correct spelling above, and explained: "What you wrote is not quite right. Look at this spelling. This is the correct spelling of the word". The Training 2 phase was repeated for that word one more time before proceeding to train the next word in the list (using the Training 1 procedure). Once all five words in a list had been trained, the instructor proceeded to Training 3.

Training 3 (spell). The instructor shuffled the five flashcards once again and said "Write down the word I say. I will not show you the word this time." The instructor randomly selected a card and read the word aloud. If the participant spelled the word correctly, the instructor provided positive feedback and proceeded to select another flashcard. If the participant made an error, the instructor retrained the participant on that word following the 'Training 2' procedure.

Training 4 (mastery). If the participant spelled all five training items correctly during 'Training 3', the set was considered "mastered", and the instructor trained a new set of items in the following session. If the participant made an error on one or more items in Training 3, then the steps outlined in Training 1, 2, and 3 were repeated for the same set in the next session. This was repeated until that list was mastered.

Training 5 (revision). At the end of each session, the instructor revised the list that was mastered in the previous session. Any words spelled incorrectly during this phase were carried forward to the Training 5 phase in the next training session. An example of the training procedure for the first five sessions is depicted in detail in Appendix E.

\section{Brain Stimulation Protocol}

tDCS was administered using a Magstim HDCkit (The Magstim Company Ltd., UK). In order to stimulate the left temporal cortex, we used the same electrode materials and placement protocol reported for Experiment 1 above. Participants in the tDCS condition received stimulation delivered to the left temporal cortex with an initial ramp-up period of 10 seconds, after which the injecting current 
reached a maximum of $1.5 \mathrm{~mA}$. The total stimulation duration was 15 minutes, with the first 5 minutes being rest, and the subsequent 10 minutes taking place during the spelling training protocol. This duration was long enough to ensure the training procedure could be completed before the stimulation protocol ended as the training instruction component of each session never lasted longer than 10 minutes. The average impedance measured at the stimulating electrode was calculated at the end of each session. This was always below $7 \mathrm{k} \Omega,(M=4.03, S D=1.90)$, in line with the manufacturer's user recommendations for optimal stimulation parameters (The Magstim Company Ltd., UK).

Participants in the sham condition received the same stimulation during the initial 10 second ramp-up period, after which no stimulation was delivered. All participants completed each of the eight training sessions at the same time of day, with all but two participants completing training sessions in the afternoon. The two participants who completed their training in the morning comprised of an individual from the tDCS group and another from the sham treatment group. As such, chronotype was matched across training sessions for all individuals, and counterbalanced across treatment groups. As for Experiment 1, at the end of each training session, all participants were asked to rate the extent to which they experienced (1) difficulty concentrating, (2) relaxation, (3) headache, (4) fatigue, (5) nervousness, (6) tingling, (7) itching, (8) burning, or (9) pain.

\section{Results}

Figure 2 shows immediate (Test 2 minus Test 1) and delayed (Test 3 minus Test 1) spelling accuracy gains for the two groups using percentage correct scores (left) and letter match scores (right). The distribution of data for all measures were normally distributed as assessed with Shapiro-Wilk tests (all $p \mathrm{~s}>.147)$, and were equivariant between groups as assessed with Levene's test (al; $p \mathrm{~s}>.583)$. 


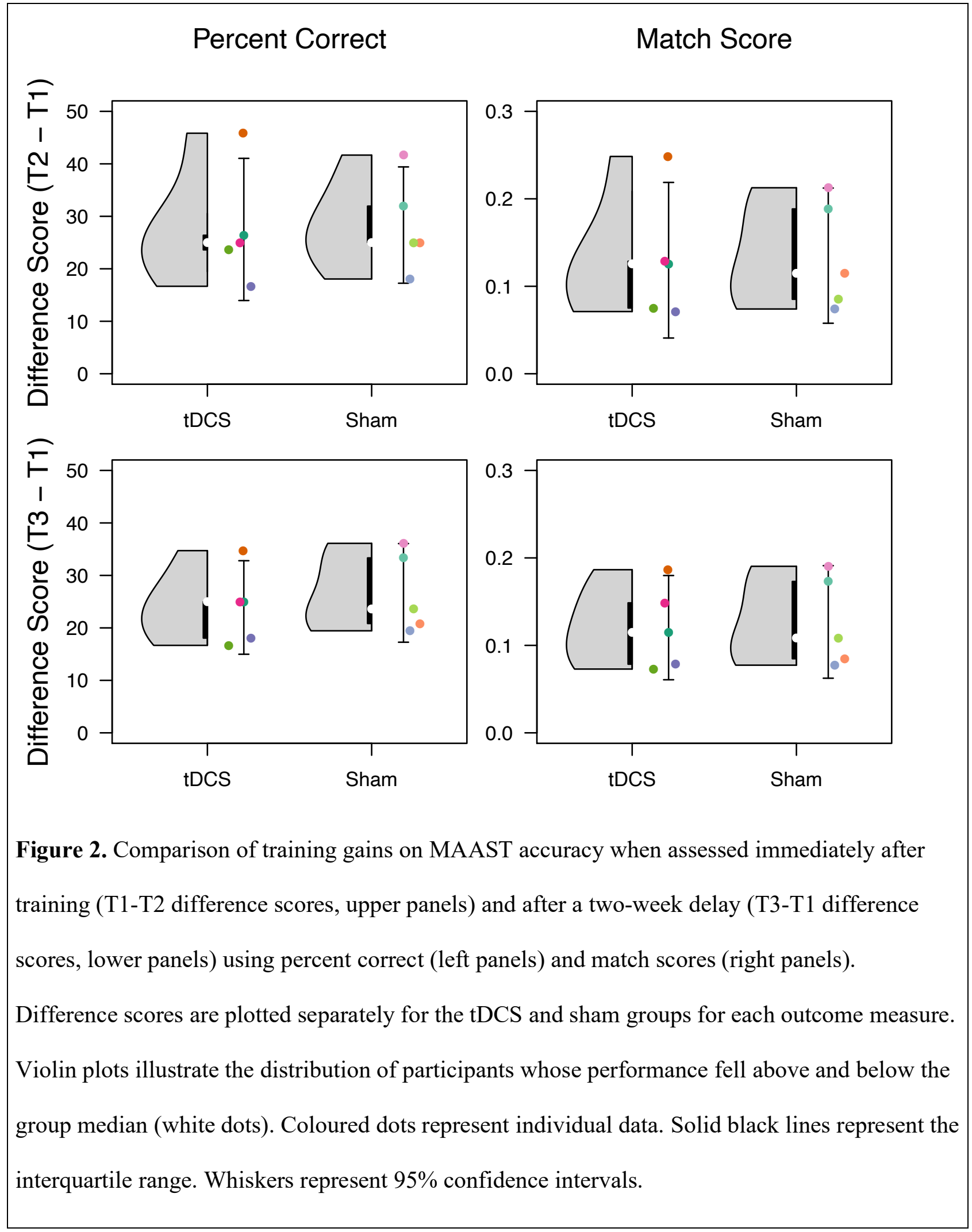


To compare the effects of tDCS versus sham on spelling accuracy percentage and match scores, we used independent samples t-tests to compare the mean difference scores between Test 1 and 2 (T2$\mathrm{T} 1$; immediate training gains) and Test 1 and 3 (T3-T1; delayed training gains) between the two groups (see Figure 3). We found no evidence for group differences on either of the immediate or delayed measures (see Table 3).

Close inspection of the individual data presents a compelling case for no effect of tDCS on enhancing spelling training effectiveness. First, the distribution of spelling training gains across both tDCS and sham groups was remarkably homogenous. Second, there appeared to be a trend of slightly better spelling training outcomes emerging in the sham group. Whilst this was not statistically significant, these data definitely indicate that there was no trend emerging for any additional benefit of tDCS. An inspection of immediate spelling training gains (i.e., T2-T1 difference scores) revealed that for all bar one participant in the tDCS group, there was a participant in the sham group who made larger spelling training gains. The one exception was a tDCS participant who demonstrated the largest improvement in spelling immediately following the training program (i.e., at T2). However, this person also exhibited the largest regression in spelling performance at delayed testing (i.e., at T3). Further inspection of longer-term spelling gains (i.e., T3-T1 difference scores) confirmed that for all participants in the tDCS group, there was a participant in the sham group who exhibited larger sustained training gains. Combined with the group analyses above, the individual data strongly suggest no real effect of tDCS on the effectiveness of spelling training.

\begin{tabular}{|c|c|c|c|c|c|c|c|c|c|c|c|c|}
\hline & T1 & & $\mathbf{T 2}$ & & T3 & & T2-T1 & & & T3-T1 & & \\
\hline & Sham & tDCS & Sham & tDCS & Sham & tDCS & Sham & tDCS & $t, p$, Cohen's d & Sham & tDCS & $t, p$, Cohen's d \\
\hline $\begin{array}{l}\text { Percent } \\
\text { correct }\end{array}$ & $\begin{array}{l}45.00 \\
(10.96)\end{array}$ & $\begin{array}{l}44.44 \\
(12.62)\end{array}$ & $\begin{array}{l}73.33 \\
(9.34)\end{array}$ & $\begin{array}{l}71.94 \\
(16.45)\end{array}$ & $\begin{array}{l}71.67 \\
(8.25)\end{array}$ & $\begin{array}{l}68.33 \\
(12.89)\end{array}$ & $\begin{array}{l}28.33 \\
(8.93)\end{array}$ & $\begin{array}{l}27.50 \\
(10.91)\end{array}$ & $.132, .898, .084$ & $\begin{array}{l}26.67 \\
(7.57)\end{array}$ & $\begin{array}{l}23.89 \\
(7.18)\end{array}$ & $.595, .568, .377$ \\
\hline Match & $\begin{array}{l}0.74 \\
(0.08)\end{array}$ & $\begin{array}{l}0.73 \\
(0.09)\end{array}$ & $\begin{array}{l}0.88 \\
(0.05)\end{array}$ & $\begin{array}{l}0.86 \\
(0.11)\end{array}$ & $\begin{array}{l}0.87 \\
(0.05)\end{array}$ & $\begin{array}{l}0.85 \\
(0.08)\end{array}$ & $\begin{array}{l}0.14 \\
(0.06)\end{array}$ & $\begin{array}{l}0.13 \\
(0.07)\end{array}$ & $.123, .905, .078$ & $\begin{array}{l}0.13 \\
(0.05)\end{array}$ & $\begin{array}{l}0.12 \\
(0.05)\end{array}$ & $.207, .841, .131$ \\
\hline
\end{tabular}

Note. Descriptive statistics are reported in the format M(SD). Comparison stats are in the format t-statistic, p-value, Cohens' d 
Given the absence of a tDCS effect on spelling training, we collapsed the data across the treatment groups to determine if the spelling training itself was effective. Spelling accuracy percentage and match scores were normally distributed (all Shapiro-Wilk $p s>1.49$ ), and so we used parametric paired t-tests to compare the mean difference scores for immediate (T2-T1) and delayed (T3-T1) spelling training effects. Both measures revealed large and statistically significant training gains (see Table 4 for descriptive and comparison statistics). Thus, the spelling training was highly effective regardless of stimulation treatment.

Table 4

Immediate and delayed training effects collapsed across stimulation condition.

\begin{tabular}{llllll}
\hline & T1 & T2 & T3 & T1 vs T2 & $\begin{array}{l}\text { T1 vs T3 } \\
\boldsymbol{t}, \boldsymbol{p}, \boldsymbol{p} \text {, Cohen's } \boldsymbol{d} \text {,s } \boldsymbol{d}\end{array}$ \\
\hline Percent Correct & $44.72(11.15)$ & $72.64(12.63)$ & $70.00(10.36)$ & $9.38,<.001^{*}, 2.97$ & $11.25,<.001^{*}, 3.56$ \\
Match Score & $0.73(0.08)$ & $0.87(0.08)$ & $0.86(0.06)$ & $6.61,<.001^{*}, 2.09$ & $8.28,<.001^{*}, 2.62$ \\
\hline
\end{tabular}

Note. Descriptive statistics are reported in the format M(SD). Comparison stats are in the format t-statistic, p-value, Cohens' d.

Finally, independent samples Mann-Whitney t-tests were used to compare participants' experience ratings across the tDCS and sham treatment groups. For each individual we calculated the average of all the ratings provided across the eight training sessions. We found no significant differences between groups for any of the rating items (all $p \mathrm{~s}>.387$ ) suggesting that participants were not aware of their respective treatment allocation (see Appendix C for relevant statistics).

\section{Discussion}

Experiment 2 failed to find any evidence for left-temporal tDCS enhancement of spelling training effects in adults for words that they could not spell accurately. The absence of a tDCS effect cannot be explain by differences in groups, who had comparable spelling, reading, spoken word retrieval, and non-verbal intelligence prior to training. Nor can this be ascribed to differences in the training paradigm, which was the same for all participants; to an ineffective training program, which 
showed large and reliable effects on spelling; or to differences in subjective experiences of tDCS or sham, which were reported to be similar by the two groups.

It might be argued that using adults with typical spelling abilities limits the potential for training-related gains to be detected. However, this argument is challenged by evidence for large spelling effects in the adults with typical spelling skills in this experiment. Alternatively, it might be argued that tDCS only enhances training effects in adolescents and adults with developmental dyslexia (Costanzo et al., 2019; Costanzo, Varuzza, Rossi, Sdoia, Varvara, Oliveri, Giacomo, et al., 2016; Heth \& Lavidor, 2015). This argument is hard to defend given that people with dyslexia are, by definition, "resistant" to adequate reading instruction, and hence respond less readily to literacy training than typical readers. Some people might then argue that people with developmental dyslexia have "atypical" brains that may respond differently to tDCS stimulation than the brain of a typical reader. However, people with developmental dyslexia do not have atypical brains. Like people with typical reading, they have brains that are arranged in a way that happens to make reading a difficult skill to acquire, just as some people have brains that make piano playing a difficult skill to acquire (Protopapas \& Parrila, 2018).

If the absence of an effect of tDCS on spelling training cannot be explained by factors related to participants, it might be explained by the way that we applied tDCS. One obvious factor is location. tDCS administration protocol requires the generation of a closed loop current which means some parts of the brain will increase in positive charge (i.e., left temporal region in this study) and some will increase in negative charge (i.e., right temporal region). Hence, the absent tDCS effect may be due to the inhibition of the right hemispheric processes which may be important for spelling (Thomson et al., 2015). Likewise, there may also be neural populations in the left hemisphere which serve an important inhibitory role during the spelling of irregular words (e.g., hypothetically suppressing graphenephoneme mappings which regularize the word). If this is true, it is possible that tDCS to the left hemisphere simultaneously boosts some excitatory information processing pathways but interferes with 
other inhibitory pathways that are equally critical for either learning (i.e., encoding) or recalling (i.e., retrieving) word spellings. Given individual differences in neural organization, it is possible that a tDCS paradigm may have an impact on some individuals but not others. It should be noted that our experiments, which were carefully designed to maximize the probability of finding a tDCS effect, failed to find any such effect in 26 individuals with typical reading and spelling. Whilst this is a relatively modest sample, our data suggests that the proportion of individuals, if any, who may benefit from any one tDCS paradigm is likely to be very small. It might be possible to increase this proportion by carefully scanning the brains of individuals to localise regions that respond to known and unknown words and then use this information to identify individually tailored regions for subsequent stimulation. However, the stimulation provided by tDCS is so diffuse that one cannot guarantee the specific activation of the target area without impacting other cortical regions. A sensible approach might be to use a more accurate technique, such as transcranial magnetic stimulation, to target specific sites.

\section{Conclusion}

Taken together, these studies provide no evidence for an effect of tDCS on reading or spelling. Experiment 1 found no difference in performance on reading and spelling tests using tDCS or sham stimulation in adults. Experiment 2 found strong evidence for a large effect of spelling training in adults, which was not enhanced by tDCS compared to sham stimulation. We were somewhat surprised by these outcomes, given that these studies were designed to maximise any potential effects of tDCS on reading or spelling. However, the results were resoundingly clear at both the group and individual level.

Our findings join a number of previous studies that have failed to find an effect of tDCS on reading or spelling skills. The collective outcomes of these studies are starting to raise red flags for researchers, such as ourselves, who try to support people with developmental difficulties. People who live with such difficulties, including parents, are vulnerable to trying unproven interventions due to (1) the direct and indirect negative consequences of living with a learning difficulty which can present 
significant motivation to seek intervention, and (2) a dearth of regulations around the provision of nonevidence-based interventions. Evidence to date suggests that people with developmental dyslexia respond best to reading and spelling training paradigms that are known to be effective for people with typical reading and spelling. Future tDCS studies should not recruit people with developmental dyslexia until there is clear evidence that tDCS has a reliable and valid effect in a non-vulnerable population.

\section{Acknowledgements}

The authors wish to acknowledge Paul Sowman for his assistance with obtaining ethics clearance for Experiment 1 and his advice on developing safe tDCS protocol. We would also like to acknowledge Nic Badcock for his feedback on experimental design for Experiment 2. Finally, we sincerely thank all of the participants for taking part.

\section{Data availability}

Research data associated with this paper can be found on the Open Science Framework. The following link can accessed via DOI 10.17605/OSF.IO/V645K

The project will be made public upon acceptance of the manuscript for publication. 


\section{References}

Aylward, E. H., Richards, T. L., Berninger, V. W., Nagy, W. E., Field, K. M., Grimme, A. C., .. . Cramer, S. C. (2003). Instructional treatment associated with changes in brain activation in children with dyslexia. Neurology, Jul 22(2), 212-219.

Bikson, M., Datta, A., \& Elwassif, M. (2009). Establishing safety limits for transcranial direct current stimulation. Clinical neurophysiology : official journal of the International Federation of Clinical Neurophysiology, 120(6), 1033-1034. doi:10.1016/j.clinph.2009.03.018

Boehringer, A., Macher, K., Dukart, J., Villringer, A., \& Pleger, B. (2013). Cerebellar Transcranial Direct Current Stimulation Modulates Verbal Working Memory. Brain Stimulation: Basic, Translational, and Clinical Research in Neuromodulation, 6(4), 649-653. doi:10.1016/j.brs.2012.10.001

Cancer, A., \& Antonietti, A. (2018). tDCS Modulatory Effect on Reading Processes: A Review of Studies on Typical Readers and Individuals With Dyslexia. Frontiers in Behavioral Neuroscience, 12, 162-162. doi:10.3389/fnbeh.2018.00162

Cao, F., Bitan, T., \& Booth, J. R. (2008). Effective brain connectivity in children with reading difficulties during phonological processing. Brain and Language, 107(2), 91-101. doi:10.1016/j.bandl.2007.12.009

Caruana, N., Colenbrander, D., \& McArthur, G. (2019). The Macquarie University Advanced Adult Spelling Test (MAAST). Macquarie Online Test Interface (MOTIf; www.motif.org.au)

Coltheart, M., Rastle, K., Perry, C., Langdon, R., \& Ziegler, J. (2001). DRC: a dual route cascaded model of visual word recognition and reading aloud. Psychological Review, 108(1), 204-256. doi:10.1037/0033-295x.108.1.204

Conrad, N. J., Kennedy, K., Saoud, W., Scallion, L., \& Hanusiak, L. (2019). Establishing word representations through reading and spelling: comparing degree of orthographic learning. Journal of Research in Reading, 42(1), 162-177. doi:10.1111/1467-9817.12256 
Costanzo, F., Rossi, S., Varuzza, C., Varvara, P., Vicari, S., \& Menghini, D. (2019). Long-lasting improvement following tDCS treatment combined with a training for reading in children and adolescents with dyslexia. Neuropsychologia, 130, 38-43.

doi:10.1016/j.neuropsychologia.2018.03.016

Costanzo, F., Varuzza, C., Rossi, S., Sdoia, S., Varvara, P., Oliveri, M., . . Menghini, D. (2016).

Evidence for reading improvement following tDCS treatment in children and adolescents with Dyslexia. Restorative Neurology And Neuroscience, 34(2), 215-226. doi:10.3233/RNN-150561

Costanzo, F., Varuzza, C., Rossi, S., Sdoia, S., Varvara, P., Oliveri, M., . . Menghini, D. (2016). Reading changes in children and adolescents with dyslexia after transcranial direct current stimulation. NeuroReport, 27, 295-300. doi:doi.org/10.1097/WNR.0000000000000536

Cummine, J., Boliek, C. A., McKibben, T., Jaswal, A., \& Joanisse, M. F. (2019). Transcranial direct current stimulation (tDCS) selectively modulates semantic information during reading. Brain and language, 188, 11-17.

Davis, C. J., \& Bowers, J. S. (2006). Contrasting five different theories of letter position coding: evidence from orthographic similarity effects. Journal of experimental psychology. Human perception and performance, 32(3), 535-557. doi:10.1037/0096-1523.32.3.535

Dehaene, S., \& Cohen, L. (2011). The unique role of the visual word form area in reading. Trends in Cognitive Sciences, 15(6), 254-262. doi:https://doi.org/10.1016/j.tics.2011.04.003

Eden, G. F., \& Zeffiro, T. A. (1998). Neural systems affected in developmental dyslexia revealed by functional neuroimaging. Neuron, 21, 279-282. doi:doi.org/10.1016/s0896-6273(00)80537-1

Fertonani, A., Ferrari, C., \& Miniussi, C. (2015). What do you feel if I apply transcranial electric stimulation? Safety, sensations and secondary induced effects. Clinical neurophysiology : official journal of the International Federation of Clinical Neurophysiology, 126(11), 21812188. doi:10.1016/j.clinph.2015.03.015 
Graves, W. W., Grabowski, T. J., Mehta, S., \& Gupta, P. (2008). The left posterior superior temporal gyrus participates specifically in accessing lexical phonology. Journal of Cognitive Neuroscience, 20(9), 1698-1710. doi:10.1162/jocn.2008.20113

Heth, I., \& Lavidor, M. (2015). Improved reading measures in adults with dyslexia following transcranial direct current stimulation treatment. Neuropsychologia, 70, 107-113. doi:https://doi.org/10.1016/j.neuropsychologia.2015.02.022

Jeffreys, H. (1961). The theory of probability. New York: OUP Oxford.

Kaufman, A. S., \& Kaufman, N. L. (2004). Kaufmann Brief Intelligence Test-Second Edition (KBIT2): Pearson.

Kohnen S, Banales E. The Macquarie University Reading Clinic Sight words intervention program. Sydney: Macquarie University; 2015.

Landi, N., Mencl, W. E., Frost, S. J., Sandak, R., \& Pugh, K. R. (2010). An fMRI study of multimodal semantic and phonological processing in reading disabled adolescents. Annals of dyslexia, 60(1), 102-121. doi:10.1007/s11881-009-0029-6

Lee, M. D., \& Wagenmakers, E. J. (2014). Bayesian cognitive modeling: A practical course. Cambridge: Cambridge University Press.

McKenna, P., \& Warrington, E. K. (1980). Testing for nominal dysphasia. Journal of Neurology, Neurosurgery \& Psychiatry, 43(9), 781. doi:10.1136/jnnp.43.9.781

Nicholls, M. E., Thomas, N. A., Loetscher, T., \& Grimshaw, G. M. (2013). The Flinders Handedness survey (FLANDERS): a brief measure of skilled hand preference. Cortex, 49(10), 2914-2926.

Nitsche, M. A., Schauenburg, A., Lang, N., Liebetanz, D., Exner, C., Paulus, W., \& Tergau, F. (2003). Facilitation of implicit motor learning by weak transcranial direct current stimulation of the primary motor cortex in the human. Journal of Cognitive Neuroscience, 15(4), 619-626. doi:10.1162/089892903321662994 
Oldfield, R. C. (1971). The assessment and analysis of handedness: The Edinburgh inventory. Neuropsychologia, 9(1), 97-113. doi:https://doi.org/10.1016/0028-3932(71)90067-4

Poreisz, C., Boros, K., Antal, A., \& Paulus, W. (2007). Safety aspects of transcranial direct current stimulation concerning healthy subjects and patients. Brain research bulletin, 72(4-6), 208-214. doi:10.1016/j.brainresbull.2007.01.004

Protopapas, A., \& Parrila, R. (2018). Is dyslexia a brain disorder?. Brain sciences, 8(4), 61.).

Pugh, K. R., Mencl, W. E., Jenner, A. R., Katz, L., Frost, S. J., Lee, J. R., . . Shaywitz, B. A. (2000). Functional neuroimaging studies of reading and reading disability (developmental dyslexia). Mental retardation and developmental disabilities research reviews, 6, 207-213. doi:10.1002/1098-2779(2000)6

Rios, D. M., Correia Rios, M., Bandeira, I. D., Queiros Campbell, F., de Carvalho Vaz, D., \& Lucena, R. (2018). Impact of transcranial direct current stimulation on reading skills of children and adolescents with dyslexia. Child neurology open, 5, 2329048X18798255.

Russo, R., Wallace, D., Fitzgerald, P. B., \& Cooper, N. R. (2013). Perception of comfort during active and sham transcranial direct current stimulation: a double blind study. Brain stimulation, 6(6), 946-951. doi:10.1016/j.brs.2013.05.009

Seidenberg, M. (2017). Language at the Speed of Sight: How we Read, Why so Many Can't, and what can be done about it. USA: Basic Books.

Simos, P. G., Fletcher, J. M., Bergman, E., Breier, J. I., Foorman, B. R., Castillo, E. M., .. . Papanicolaou, A. C. (2002). Dyslexia-specific brain activation profile becomes normal following successful remedial training. Neurology, 58, 1203-1213. doi:doi.org/10.1212/wnl.58.8.1203

Temple, E., Poldrack, R. A., Protopapas, A., Nagarajan, S., Salz, T., Tallal, P., . . Gabrieli, J. D. E. (2000). Disruption of the neural response to rapid acoustic stimuli in dyslexia: Evidence from functional MRI. Proceedings of the National Academy of Sciences, 97, 13907-13912. 
Thomson, J. M., Doruk, D., Mascio, B., Fregni, F., \& Cerruti, C. (2015). Transcranial direct current stimulation modulates efficiency of reading processes. Frontiers in Human Neuroscience, 9(114). doi:10.3389/fnhum.2015.00114

Torgesen, J. K., Wagner, R. K., \& Rashotte, C. A. (1999). Test of Word Reading Efficiency (TOWRE). Austin: PRO-ED.

Torgesen, J. K., Wagner, R. K., \& Rashotte, C. A. (2012). Test of Word Reading Efficiency (2nd ed.). Austin, TX: Pro-Ed.

Tsapkini, K., Vindiola, M., \& Rapp, B. (2011). Patterns of brain reorganization subsequent to left fusiform damage: fMRI evidence from visual processing of words and pseudowords, faces and objects. NeuroImage, 55(3), 1357-1372. doi: https://doi.org/10.1016/j.neuroimage.2010.12.024

Turkeltaub, P. E., Benson, J., Hamilton, R. H., Datta, A., Bikson, M., \& Coslett, H. B. (2012). Left lateralizing transcranial direct current stimulation improves reading efficiency. Brain stimulation, 5(3), 201-207. doi:10.1016/j.brs.2011.04.002

Welcome, S. E., \& Joanisse, M. F. (2012). Individual differences in skilled adult readers reveal dissociable patterns of neural activity associated with component processes of reading. Brain and Language, 120(3), 360-371. doi: https://doi.org/10.1016/j.bandl.2011.12.011

Westwood, S. J., Olson, A., Miall, R. C., Nappo, R., \& Romani, C. (2017). Limits to tDCS effects in language: Failures to modulate word production in healthy participants with frontal or temporal tDCS. Cortex, 86, 64-82. doi:10.1016/j.cortex.2016.10.016

Wilkinson, G. S., \& Robertson, G. J. (2006). Wide Range Achievement Test 4 Professional Manual. Lutz, FL: Psychological Assessment Resources.

Wilson, J. K. (2018). Transcranial Direct Current Stimulation (tDCS) Improves Performance on Spelling and Word Detection Tasks. Retrieved from https://digitalrepository.unm.edu/psy_etds/247 
Younger, J. W., \& Booth, J. R. (2018). Parietotemporal Stimulation Affects Acquisition of Novel Grapheme-Phoneme Mappings in Adult Readers. Frontiers in Human Neuroscience, 12(109). doi:10.3389/fnhum.2018.00109

Younger, J. W., Randazzo Wagner, M., \& Booth, J. R. (2016). Weighing the Cost and Benefit of Transcranial Direct Current Stimulation on Different Reading Subskills. Frontiers in Neuroscience, 10(262). doi:10.3389/fnins.2016.00262 